\title{
Transoral Resection of a Retropharyngeal Myxoma: A Case Report
}

\author{
James R. White, B.Sc.,' Sean Weiss, M.D.,' 1 Dwayne Anderson, M.D. ${ }^{2}$ \\ Stephen E. Mason, M.D., ${ }^{3}$ Mell A. Schexnaildre, M.A., S.S.C.-S.L.P., 1,4 \\ Daniel W. Nuss, M.D., ${ }^{1}$ and Rohan R. Walvekar, M.D.' ${ }^{1}$
}

This study was conducted to describe a retropharyngeal myxoma and discuss clinical concerns regarding this pathology and a retropharyngeal site of occurrence. We present a case report and review of literature. A 71-year-old woman presented with mild right neck pressure for 3 weeks. Imaging studies and head neck examination confirmed a $5.3 \times 3.1 \times 1.0 \mathrm{~cm}$ retropharyngeal mass with no communication to the vertebral column but was intimately involved with the pharyngeal mucosa. A transoral fine needle aspiration biopsy suggested a possible spindle cell neoplasm. A presurgical swallowing consultation was obtained. A transoral excision of the tumor was possible with no intraoperative complications. Histopathology was a cellular myxoma. Postoperative dysphagia required swallowing therapy and nasogastric tube feeding for 2 weeks before oral intake was possible. The patient has no evidence of clinical or radiological recurrence more than 1 year after surgical intervention. We present the second case of a myxoma in the retropharynx reported in English literature. Transoral excision was safe, feasible, and cosmetically appealing option in our patient. Additional clinical data are required to valid its safety and utility as an approach to tumors in the retropharynx. Postoperative dysphagia can be significant and consequently we recommend preoperative swallowing evaluation and counseling.

KEYWORDS: Retropharyngeal, myxoma, transoral surgery, retropharynx

$M_{\text {yxomas are benign, locally infiltrative neo- }}$ plasms arising from mesenchymal origin. ${ }^{1}$ They are typically observed in myocardium, ${ }^{2}$ but can also arise from bone and other soft tissues. ${ }^{1}$ Myxomas are rarely seen in the head and neck region and are most often found to arise from bone. Soft tissue myxomas of the head and neck are even more uncommon. ${ }^{3}$ Pahor and Samant (1994) found only 60 cases of soft tissue myxomas of the head and neck reported in the literature. ${ }^{4}$ Since that study, there have been only 19 additional cases reported to date ${ }^{5-16}$ (Table 1). In their study, Pahor and Samant presented the first case of a retropharyngeal soft tissue myxoma managed with a transcervical approach for resection. ${ }^{4}$ We present the first case of a retropharyngeal myxoma excised by way of a transoral approach and a literature review on head and neck myxomas updated since $1994^{3}$
${ }^{1}$ Department of Otolaryngology Head Neck Surgery, LSU Health Sciences Center, New Orleans; ${ }^{2}$ Department of Neuroradiology and Interventional Neuroradiology; ${ }^{3}$ Pathology Group of Louisiana; ${ }^{4}$ Voice Center, Our Lady of the Lake Regional Medical Center, Baton Rouge, Louisiana.

Address for correspondence and reprint requests: Rohan $\mathrm{R}$. Walvekar, M.D., Assistant Professor, Department of Otolaryngology Head Neck Surgery, LSU Health Sciences Center, 533 Bolivar Street,
Suite 566, New Orleans, LA 70112 (e-mail: rwalve@1suhsc.edu). Skull Base Rep 2011;1:139-144. Copyright (C) 2011 by Thieme Medical Publishers, Inc., 333 Seventh Avenue, New York, NY 10001, USA. Tel: +1(212) 584-4662.

Received: December 14, 2010. Accepted after revision: June 17, 2011. Published online: October 4, 2011.

DOI: http://dx.doi.org/10.1055/s-0031-1287685.

ISSN 2157-6971. 
Table 1 Soft Tissue Myxomas of the Head and Neck: Updated Review ${ }^{5-16}$

\begin{tabular}{ll}
\hline Location & No. of Cases \\
\hline Palate & 10 \\
Larynx & 10 \\
Parotid & 6 \\
Lateral neck & 6 \\
Oral & 5 \\
Scalp & 4 \\
Lip & 4 \\
Oropharynx & 4 \\
Cheek & 3 \\
Masseter & 3 \\
Alveolar ridge & 2 \\
Face & 2 \\
Temporalis & 2 \\
Retropharyngeal & $\mathbf{2}$ \\
Chin & 1 \\
Tongue & 1 \\
Conjunctiva & 1 \\
Buccal mucosa & 1 \\
Interdental gingiva & 1 \\
Retromolar pad & 1 \\
Geniohyoid muscle & 1 \\
Eyelid & 1 \\
External auditory canal & 1 \\
Laryngopharynx & 1 \\
Cornea & 1 \\
Infratemporal fossa & 1 \\
Nasal vestibule & 1 \\
Scalene muscle & 1 \\
Posterior neck & 1 \\
Posterolateral neck & 1 \\
Total & 1 \\
\hline & 1 \\
\hline
\end{tabular}

\section{CASE REPORT}

In October of 2009, a 71-year-old woman was referred to our service at Our Lady of the Lake Regional Medical Center in Baton Rouge, LA, and to the Department of Otolaryngology Head \& Neck Surgery, Louisiana State University Health Sciences Center, New Orleans, LA, with a chief complaint of right-sided neck pressure for 2 to 3 weeks. The patient reported having no dysphagia, odynophagia, dyspnea, change of voice, but did admit to a sore throat in addition to the neck pressure. She denied fevers, weight loss, or a recent history of infections. Her medical history was significant for non-Hodgkin lymphoma treated with chemotherapy for 2 years before undergoing a stem cell transplant in 2004. She has been in remission since then. Physical examination revealed a lesion in the retropharynx that created a bulge in the intact mucosa of the pharynx that extended from the nasopharynx to the mid-oropharynx on the right via flexible laryngoscopy. The lesion did not appear to cross the midline and as mentioned previously, the pharyngeal mucosa overlying the lesion was intact (Fig. 1). There was no vocal fold involvement and all cranial nerves were intact. A computed tomography (CT) scan revealed a possible cystic mass in the right oropharynx. However, a subsequent magnetic resonance imaging (MRI) of the neck with and without contrast clearly revealed a $5.3 \mathrm{~cm} \times 3.1 \mathrm{~cm} \times 1.0 \mathrm{~cm}$ well-circumscribed mass centered in the right retropharyngeal space. The mass demonstrated atypical signal characteristics; in that it followed simple fluid on T2-weighted sequences while it uniformly enhanced on postgadolinium sequences, (Fig. 2A, B). A positron emission tomography (PET) and CT fusion scan obtained for lymphoma restaging revealed a low level hypermetabolism in a low-density soft tissue mass in the right retropharyngeal space with a

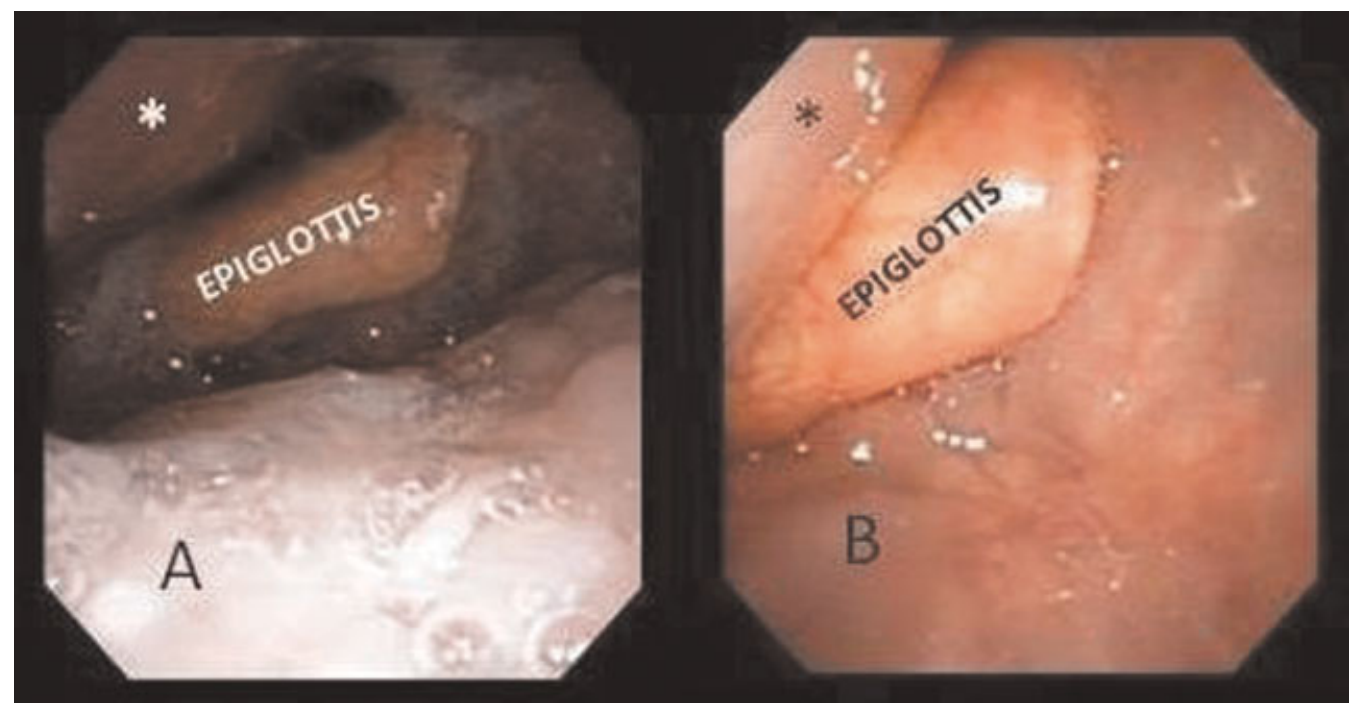

Figure 1 A flexible fiberoptic laryngoscopy view of the retropharyngeal mass. (A) Depicts the mass at presentation in the right retropharynx. (B) Shows the mass after a period of observation with an increase in size and now touching the epiglottis. The asterisk $\left({ }^{*}\right)$ shows the right retropharyngeal mass. 

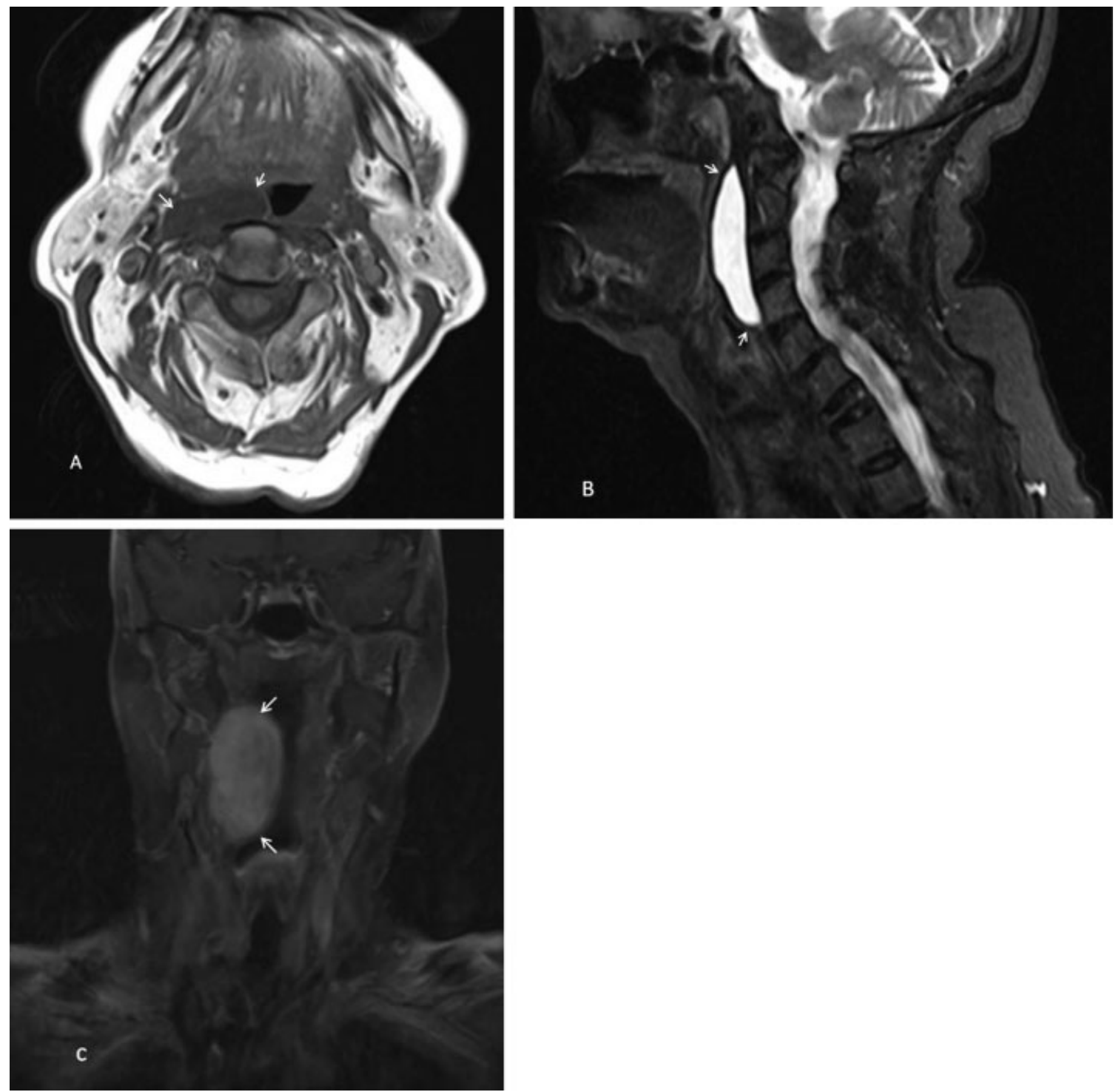

Figure 2 (A) T1-axial noncontrast MR image showing the tumor in axial view showing its close proximity to carotid sheath (white arrows define tumor extent). (B) T2-sagittal MR image showing retropharyngeal site of the mass and superior-inferior extent (white arrows define tumor extent). (C) T1-postcontrast fat saturated coronal MR image showing protrusion of the tumor into the pharynx (white arrows define tumor extent).

maximum standard uptake value of 2.2. The scan did not appear to have significantly changed when compared with the report from a prior PET scan performed in May of 2008. A subsequent fine needle aspiration biopsy revealed groups of bland spindle cells with fibrillary cytoplasmic processes. The needle biopsy was performed transorally as the tumor was easily accessible through the mouth as an alternative to more conventional transcervical imaged-guided needle biopsy. There was no evidence of malignancy or lymphoma. The patient could not have treatment at this time due to personal reasons. A follow-up evaluation in 3 months, demonstrated a marginal increase in tumor size in an inferior direction. The tumor was now abutting the epiglottis. However, the patient continued to be mainly asymptomatic.
Treatment options that included observation and surgery were discussed. Observation is often a reasonable alternative to surgery given that these tumors are slow growing and surgical resection of advanced tumors can be challenging in terms of achieving clear margins, and the associated risk of morbidity such as postoperative swallowing dysfunction. In view of the fact that tumor had increased in size, the patient opted for surgical excision via a transcervical or a possible transoral route. A presurgical swallowing consultation was requested to counsel the patient regarding the possibility of postoperative swallowing dysfunction associated with interruption of pharyngeal plexus and musculature as a consequence of the surgical approach to the tumor. A transoral resection of the tumor was performed without 
any intraoperative complications. Surgical exposure was assessed using a tonsil mouth retractor. The tumor was found to be mobile over the prevertebral muscles. The superior limit of the tumor could be exposed with transoral palatal retraction. The inferior and lateral limits of the tumor were also accessible on intraoperative evaluation. Consequently, we proceeded with transoral tumor removal that was performed using sharp dissection techniques and the use of an extended-tip cautery. Hemostasis was achieved with a bipolar cautery. Intraoperatively, the tumor was found to be anterior to the prevertebral fascia within the confines of the retropharyngeal space. However, the tumor was not completely encapsulated and had components that were intertwined with the pharyngeal musculature. The dissection required exposure of the carotid artery laterally. Pathologic evaluation revealed the mass to be a cellular myxoma of soft tissue origin (Fig. 3).

Postoperatively, the patient had significant dysphagia secondary to pharyngeal muscle dysfunction evident upon fluoroscopic studies and endoscopic evaluation. She required aggressive postoperative swallowing therapy and nasogastric tube feeding for 2 weeks before regular oral intake was possible. A postoperative CT scan and MRI scan done at 4 and 8 months after surgery revealed no evidence of recurrence. The patient continues to do well with no evidence of local recurrence. The patient also denoted normal swallowing function that was confirmed by postoperative fluoroscopic studies and endoscopic evaluation.

\section{DISCUSSION}

Myxomas are benign mesenchymal neoplasms that consist of a loose mucoid stroma and undifferentiated stellate cells. They are composed of an abundant mucopolysaccharide matrix, and have little vascularity, cellularity, or cytological atypia ${ }^{5}$ (Fig. 3). In 1871, Virchow likened these histological characteristics to that of the mucinous tissue of the umbilical cord, and he was the first to use the term "myxoma" to describe such a neoplasm. ${ }^{17}$ This gelatinous quality gives the myxoma a firm, rubbery consistency. ${ }^{18}$ While their exact etiology remains unclear, most researchers agree that altered fibroblasts begin secreting glycosaminoglycans in excess. ${ }^{19,20}$ Inciting events have been thought to be infection and trauma. ${ }^{5}$

In general, myxomas are rare entities, comprising only $0.12 \%$ of all soft tissue tumors. They have also been documented to have an overall occurrence of only 0.13 per 100,000 individuals. ${ }^{10}$ Myxomas of the head and neck are even more rare and occur most frequently in the myocardium. ${ }^{2}$ Andrews et al (2000) reported 169 myxomas of the head and neck region and found $61 \%$ originated in bone and most frequently involved the mandible or maxilla (76\%). Soft tissue myxomas were more variably located. ${ }^{1}$

Myxomas are slow growing and remain asymptomatic for years. ${ }^{1}$ Their indolent growth typically causes patients to present with a painless mass, unless they extend into adjacent structures. While these tumors do not metastasize, they are locally infiltrative in nature. Rarely they may cause fatal infiltration and involvement of anatomic structures like the trachea and esophagus. ${ }^{21}$ Myxomas lack of a complete fibrous capsule that contributes to their characteristic local infiltration into fat and muscle and increases their potential for recurrence. When striated muscle is involved, there may be a surprising degree of interdigitation with the musculature causing destruction of the tissue and/or significant postoperative morbidity after surgical resection, as seen in our case. ${ }^{1}$ In addition, the gelatinous nature of a myxoma makes complete resection difficult. Consequently, longterm follow-up is recommended to monitor recurrence. $^{18}$

Myxomas are generally insensitive to radiation. Surgical resection with adequate margins is the treatment of choice. ${ }^{18}$ More conservative surgical approaches
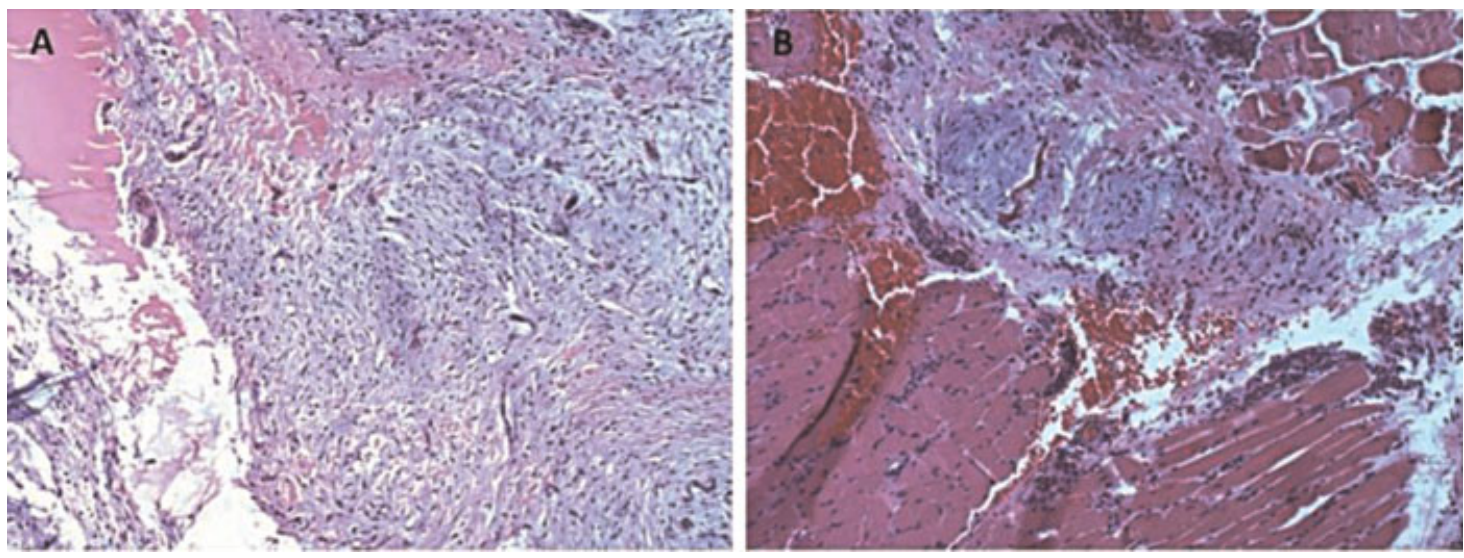

Figure 3 (A) Bland, cellular spindle cell tumor with fusiform cells with scant eosinophilic cytoplasm in a background of abundant mucopolysaccharide matrix (H\&E 100x). (B) Fragment of cellular myxoma (center) intermixed with skeletal muscle (H\&E 100x). 
include curettage and enucleation. ${ }^{22}$ Recurrence is most commonly seen with conservative surgery $(28 \%)$ and is $6 \%$ with aggressive surgical resection with clear margins. ${ }^{1}$ Conservative approaches are especially suited for lesions in close proximity to vital structures where aggressive resections are either not technically feasible or are expected to be associated with significant morbidity or mortality. ${ }^{1}$

Numerous surgical approaches to tumors of the retropharyngeal and parapharyngeal spaces have been discussed in the literature. ${ }^{23-26}$ The most common are transcervical, transparotid, combined transcervicaltransparotid, transcervical with a mandibulotomy or partial mandibulectomy, and transoral. More aggressive approaches such as a double mandibulotomy with coronoidectomy ${ }^{25}$ may be required to approach larger tumors in the lateral parapharyngeal space. Tumors of the medial parapharyngeal space and retropharyngeal space can be safely and adequately treated via the transoral approach. ${ }^{23,26}$ The transoral approach to the lesions of the retropharynx has also been successfully utilized in several circumstances and has been shown to be safe with decreased morbidity compared with traditional approaches. ${ }^{23,26-30}$ There are several surgical techniques that have been utilized for transoral tumor access such as transpalatal approach for superior tumor access and the use of a mouth retractor such as in our case. More recently, the microscope and robotic technology have added enhanced magnification, visualization, and dexterity within the posterior oral cavity and oropharynx. The addition of these technological advances will enhance exposure and access to the retropharyngeal tumors as well. Supporters of transcervical approaches to tumors of the parapharyngeal space contend that the transoral approach is contraindicated due to higher risk of hemorrhage from potential vascular tumors, damage to vessels and nerves, and the possibility of tumor implantation due to poor access via this approach. ${ }^{24}$

We believe that with better imaging techniques to delineate relevant vasculature, ability to use endoscopic or robotic units to improve visualization and control of hemorrhage, as well as the potential use of navigation system have further increased our ability to access lesions transorally safely. We agree with previous observations that are based on limited experiences that the transoral route can be a safe and a cosmetically appealing alternative to transcervical approaches to the retropharynx. However, the type of surgical approach and treatment should be individualized based on patient characteristics, tumor pathology, tumor extent, and risk to major vessels based on imaging studies.

While our patient did have dysphagia in the postoperative period, she regained complete function after conservative measures and speech and swallowing therapy. With a knowledge that soft tissue myxomas of the retropharynx tend to be intimately involved with the pharyngeal musculature, patients should be counseled preoperatively that dysphagia is to be expected and that postoperative speech and swallow therapy may be necessary regardless of the surgical approach while managing myxomas in the retropharynx.

\section{CONCLUSION}

We present the second case of a myxoma in the retropharynx reported in English literature. Although the conventional transcervical approach would have been possible for this tumor, we found that the transoral route offered a safe, feasible, and cosmetically appealing option in this case. Postoperative dysphagia can be significant due to the tendency of myxomas to be intertwined with the retropharyngeal musculature and consequently we recommend preoperative swallowing evaluation and counseling prior to surgical therapy.

\section{ACKNOWLEDGMENT}

The authors would like to thank the Department of Otolaryngology Head and Neck Surgery, Louisiana State University Health Sciences Center, New Orleans, LA.

\section{REFERENCES}

1. Andrews T, Kountakis SE, Maillard AA. Myxomas of the head and neck. Am J Otolaryngol 2000;21(3):184-189

2. Ghosh BC, Huvos AG, Gerold FP, Miller TR. Myxoma of the jaw bones. Cancer 1973;31(1):237-240

3. Canalis RF, Smith GA, Konrad HR. Myxomas of the head and neck. Arch Otolaryngol 1976;102(5):300-305

4. Pahor AL, Samant S. Retropharyngeal myxoma: a case report. J Laryngol Otol 1994;108(8):690-692

5. Nakamura A, Iguchi H, Kusuki M, Yamane H, Matsuda M, Osako S. Laryngeal myxoma. Acta Otolaryngol 2008;128(1): 110-112

6. Galera-Ruiz H, Martin-Gomez R, Esteban-Ortega F, Congregado-Loscertales M, Garcia Escudero A. Extramuscular soft tissue myxoma of the lateral neck. Rev Laryngol Otol Rhinol (Bord) 2001;122(4):259-261

7. Yoon SH, Park SH. A study of 77 cases of surgically excised scalp and skull masses in pediatric patients. Childs Nerv Syst 2008;24(4):459-465

8. Ayache S, Chatelain D, Tramier B, Strunski V. Oropharyngeal and hypopharyngeal myxoma: case report and literature review. J Laryngol Otol 2007;121(5):e5

9. Papadogeorgakis N, Petsinis V, Nikitakis N, Goutzanis L, Alexandridis C. Intramuscular myxoma of the masseter muscle. A case report. Oral Maxillofac Surg 2009;13(1): $37-40$

10. Robin C, Bastidas JA, Boguslaw B. Case report: Myxoma of the temporalis muscle. Oral Surg Oral Med Oral Pathol Oral Radiol Endod 2004;97(5):620-624

11. Serrat A, Verrier A, Espeso A, Martín J. Intramuscular myxoma of the temporalis muscle. J Oral Maxillofac Surg 1998;56(10):1206-1208 
12. Yoshida K, Kawase T, Tomita T, et al. Surgical strategy for tumors located in or extending from the intracranial space to the infratemporal fossa-advantages of the transcranial approach (zygomatic infratemporal fossa approach) and the indications for a combined transcranial and transcervical approach-. Neurol Med Chir (Tokyo) 2009;49(12):580-586

13. Patsiaoura K, Anagnostou E, Benis N. Intramuscular myxoma of the nasal vestibule. Auris Nasus Larynx 2010; 37(1):100-102

14. Ozawa H, Fujii M, Tomita T, Ogawa K. Intramuscular myxoma of scalene muscle: a case report. Auris Nasus Larynx 2004;31(3):319-322

15. Ishoo E. Intramuscular myxoma presenting as a rare posterior neck mass in a young child: case report and literature review. Arch Otolaryngol Head Neck Surg 2007;133(4):398-401

16. Crankson SJ, Al Namshan M, Al Mane K, Bamefleh H. Intramuscular myxoma: a rare neck mass in a child. Pediatr Radiol 2002;32(2):120-122

17. Virchow R. Die Cellularpatholgie in Ihrer Begründung auf Physiologische und Pathologische Gewebelehre. Berlin: August Hirschwald; 1871:563

18. Hsieh DL, Tseng HM, Young YH. Audiovestibular evolution in a patient undergoing surgical resection of a temporal bone myxoma. Eur Arch Otorhinolaryngol 2006;263(7):614-617

19. Enzinger FM. Intramuscular myxoma. Am J Clin Pathol 1965;43:104-113

20. Batsakis JG. Myxomas of soft tissues and the facial skeleton. Ann Otol Rhinol Laryngol 1987;96(5):618-619

21. Stout AP. Myxoma, the tumor of primitive mesenchyme. Ann Surg 1948;127(4):706-719
22. DeFatta RJ, Verret DJ, Ducic Y, Carrick K. Giant myxomas of the maxillofacial skeleton and skull base. Otolaryngol Head Neck Surg 2006;134(6):931-935

23. Ducic Y, Oxford L, Pontius AT. Transoral approach to the superomedial parapharyngeal space. Otolaryngol Head Neck Surg 2006;134(3):466-470

24. Stell PM, Mansfield AO, Stoney PJ. Surgical approaches to tumors of the parapharyngeal space. Am J Otolaryngol 1985; 6(2):92-97

25. Lazaridis N, Antoniades K. Condylotomy or vertical subsigmoid osteotomy with a mandibulotomy anterior to the mental foramen for improved access to the parapharyngeal space tumors. J Oral Maxillofac Surg 2008;66(3):597606

26. Le TD, Cohen JI. Transoral approach to removal of the retropharyngeal lymph nodes in well-differentiated thyroid cancer. Laryngoscope 2007;117(7):1155-1158

27. Shellenberger T, Fornage B, Ginsberg L, Clayman GL. Transoral resection of thyroid cancer metastasis to lateral retropharyngeal nodes. Head Neck 2007;29(3):258-266

28. Laccourreye L, Breheret R, Rohmer V, Dubin J, Bizon A. [Transoral resection of thyroid cancer metastasis to retropharyngeal lymph node]. Ann Otolaryngol Chir Cervicofac 2008;125(6):309-312

29. Boricić I, Stojsić Z, Mikić A, Brasanac D, Tomanović N, Bacetić D. Intramuscular hemangioma of the retropharyngeal space. Vojnosanit Pregl 2007;64(7):485-488

30. Hsieh CY, Hsiao JK, Wang CP. Retropharyngeal schwannoma excised through a transoral approach: a case report. Kaohsiung J Med Sci 2006;22(9):465-469 